\title{
Atribuições dos profissionais fisioterapeuta e educador físico no atendimento home care de pacientes pós Covid-19
}

\author{
Analysis of the attributions of physical therapists and physical educators in the home care of \\ patients after Covid-19 \\ Análisis de las atribuciones de los profesionales fisioterapeutas y educadores físicos en la atención \\ domiciliaria de los pacientes después de Covid-19
}

\begin{abstract}
Resumo
A doença por coronavírus no qual afetou a população mundial, teve um grande índice de recuperados em 2021, de acordo com os dados do ministério da saúde. As sequelas que acometem os recuperados envolvem principalmente dispneia, fadiga muscular, dificuldades neurocognitivas e consequentemente diminuição do condicionamento físico. Após o período hospitalar, é necessário que os pacientes busquem profissionais para tratamento, onde teve grande destaque o atendimento home-care. $\mathrm{O}$ acompanhamento multidisciplinar é de extrema importância, onde o objetivo desta revisão é apresentar as contribuições e diferenças no atendimento domiciliar de paciente pós Covid-19 dos fisioterapeutas e educadores físicos, que apesar de terem semelhanças quanto a utilização de exercícios físicos em seus atendimentos, os objetivos terapêuticos são divergentes, entretanto, ambos contribuem de forma significativa nas etapas de reabilitação domiciliar. Os conselhos federais são os que determinam as atribuições de cada um no tratamento dessa patologia, pois apesar de ainda estar em processo os estudos envolvendo o vírus SARS-CoV-2, existe as comprovações dos benefícios proporcionados por cada profissional na fase pós hospitalar.
\end{abstract}

Palavras-chave: Fisioterapia; Educação física; Covid-19; Atendimento domiciliar.

\begin{abstract}
The coronavirus disease that has affected the world population had a high rate of recoveries in 2021, according to data from the Ministry of Health. The sequelae that affect the recovered patients mainly involve dyspnea, muscle fatigue, neurocognitive difficulties, and consequently a decrease in physical conditioning. After the hospital period, it is necessary that the patients seek professionals for treatment, where home-care has had great prominence. The multidisciplinary follow-up is extremely important, where the objective of this review is to present the contributions and differences in the home care of patients after Covid-19 of physical therapists and physical educators, who despite having similarities regarding the use of physical exercises in their care, the therapeutic goals are divergent, however, both contribute significantly in the stages of home rehabilitation. The federal councils are the ones that determine the attributions of each one in the treatment of this pathology, because although the studies involving the SARS-CoV-2 virus are still in process, there is evidence of the benefits provided by each professional in the post-hospital phase.
\end{abstract}

Keywords: Physiotherapy; Physical education; Covid-19; Home care.

\section{Resumen}

La enfermedad del coronavirus, que ha afectado a la población mundial, tuvo una alta tasa de recuperaciones en 2021, según datos del Ministerio de Sanidad. Las secuelas que afectan a los pacientes recuperados son principalmente la disnea, la fatiga muscular, las dificultades neurocognitivas y, en consecuencia, la reducción de la aptitud física. Tras el periodo hospitalario, es necesario que los pacientes busquen profesionales para su tratamiento, donde el servicio de atención domiciliaria tuvo gran protagonismo. El seguimiento multidisciplinar es sumamente importante, donde el objetivo de esta revisión es presentar las aportaciones y diferencias en la atención domiciliaria de los pacientes tras la Covid-19 de fisioterapeutas y educadores físicos, que a pesar de tener similitudes en cuanto al uso de ejercicios físicos en su atención, los objetivos terapéuticos son divergentes, sin embargo, ambos contribuyen de forma significativa en las etapas de rehabilitación domiciliaria. Los consejos federales son los que determinan las atribuciones de cada uno 
en el tratamiento de esta patología, ya que aunque los estudios sobre el virus del SARS-CoV-2 aún están en proceso, existen evidencias de los beneficios aportados por cada profesional en la fase post-hospitalaria.

Palabras clave: Fisioterapia; Educación física; Covid-19; Atención domiciliaria.

\section{Introdução}

A doença por coronavírus (Covid-19) é uma doença infecciosa causada pelo vírus SARS-CoV-2. (OMS, 2021). Até o final da Semana Epidemiológica (SE) 35 de 2021, no dia 4 de setembro de 2021, foram confirmados 220.282.354 casos de Covid-19 no mundo. Os Estados Unidos foram o país com o maior número de casos acumulados (39.906.426), seguido pela Índia (32.988.673), Brasil (20.877.864), Reino Unido (6.973.995) e França (6.910.865). (Brasil, 2021)

Em relação às análises acerca do número de pessoas infectadas por Covid-19 no mundo e que se recuperaram, até o final da semana epidemiológica 35, estima-se que 92,7\% (204.111.385/220.282.354) das pessoas infectadas por Covid-19 no mundo se recuperaram (Brasil, 2021). Como em muitas outras doenças críticas, pacientes pós-Covid-19 podem apresentar dispneia e fadiga em repouso e durante atividades de vida diária (AVD), disfunção muscular periférica e intolerância ao exercício, além de aumento no risco de desordem pós-traumática, ansiedade e depressão. (Gastaldi, 2021)

Analisando as disfunções causadas por essa patologia e os diversos casos existentes, o Conselho Federal de Fisioterapia e Terapia Ocupacional (COFFITO) determina que "O Fisioterapeuta ou Terapeuta Ocupacional tem autonomia e independência para determinar quais pacientes ou casos podem ser atendidos ou acompanhados a distância, tal decisão deve basear-se em evidências científicas no benefício e na segurança de seus pacientes.", com isso, vemos que a fisioterapia pode atuar na reabilitação das disfunções ocasionadas pelo Covid-19 tanto na modalidade remota como na presencial no âmbito domiciliar.

Os profissionais de Educação Física também podem contribuir com a recuperação das sequelas dessa patologia, pois conforme determina o Conselho Federal de Educação Física (CONFEF): “O Profissional de Educação Física é especialista em atividades físicas, nas suas diversas manifestações, observados os preceitos de responsabilidade, segurança, qualidade técnica e ética no atendimento individual e coletivo."

Com isso, o presente estudo busca apontar as contribuições das profissões de fisioterapia e educação física no atendimento de pacientes home care pós Covid-19 e identificar suas principais diferenças, além de evidenciar as atribuições e as permissões impostas pelos conselhos federais para que o processo de reabilitação seja feito de forma adequada, tendo como público alvo pacientes adultos.

\section{Metodologia}

O estudo trata-se de uma revisão sistemática, descritiva e explicativa onde, foi realizado buscas através das plataformas Brasil Scientific Electronic Library Online (SCIELO), Google Acadêmico, PUBMED, Literatura LatinoAmericana e do Caribe em Ciências da Saúde (LILACS) e Physiotherapy Evidence Database (PEDRO), onde a busca foi limitada em artigos do ano de 2018 até 2021, com as seguintes delimitações para o critério de amostras: fisioterapia, educação física e atendimento domiciliar em português. Physioterapy, education physical, Covid-19 e home care em inglês.

Os critérios de inclusão adotados foram: caracterizar tratamento fisioterapêutico no âmbito domiciliar, principais intervenções fisioterapêuticas para atendimento dos pacientes pós-Covid-19, prática de exercícios físicos domiciliar, benefícios e contribuições da educação física em pacientes com Covid-19. Assim, foram considerados todos os artigos que se relacionavam com o perfil de paciente avaliado e direcionavam ao atendimento domiciliar. Logo, os títulos e resumo foram 
lidos para verificar se associavam com o tema em questão. Foram selecionados 7 artigos através das plataformas de buscas no qual direcionavam de forma objetiva ao conteúdo avaliado.

Foram descartados os artigos que direcionavam a atuação fisioterapêutica ambulatorial e na unidade de terapia intensiva, sobre a prática de exercícios físicos direcionados a outros perfis de pacientes e atuação da educação física em cenários diferente do domiciliar.

A análise de dados e da legislação vigente para as profissões foram feitas no Ministério da Saúde, Organização Mundial da Saúde, Conselho Federal de Fisioterapia e Terapia Ocupacional e Conselho Federal de Educação Física e Associação Brasileira de Fisioterapia Cardiorrespiratória e Fisioterapia em Terapia Intensiva.

Figura 1 - Fluxograma do processo de seleção dos artigos nas bases de dados.

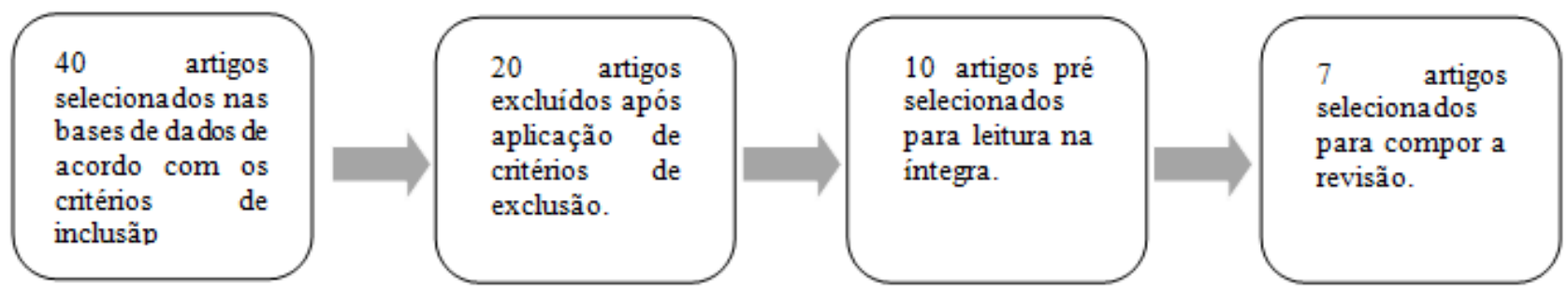

Fonte: Autores.

\section{Resultados}

Nas análises realizadas, foram encontrados apontamentos quanto a contribuição de ambas as profissões de formas distintas no atendimento domiciliar. O profissional de educação física tem como objetivo e atribuição promover melhor qualidade de vida através da execução de exercícios físicos, analisando a execução correta pelo paciente e melhorando o condicionamento físico, o mesmo não é autorizado a utilizar recursos não invasivos para auxiliar nos atendimentos, enquanto o profissional de fisioterapia tem o objetivo tanto de promover qualidade de vida como reabilitar em caso de acometimentos por patologias, onde é autorizado a utilizar recursos de ventilação não invasiva dependendo da sua avaliação, conforme sinalizado em Quadro 1.

Os profissionais destacados nesta revisão, podem trabalhar em conjunto para melhor reabilitação de paciente com os acometimentos pós Covid-19, sendo de responsabilidade do fisioterapeuta a reabilitação para posteriormente o educador físico manter a o condicionamento e bem-estar desse paciente. De acordo com as permissões estabelecidas pelos conselhos federais, vemos o porcentual de responsabilidade destacado na Figura 2 analisando os dados do Quadro 1.

Quadro 1 - Atividades do atendimento home de paciente pós Covid-19.

\begin{tabular}{|c|c|c|c|}
\hline Item & Descrição & Fisioterapia & Educação Física \\
\hline 1 & $\begin{array}{c}\text { Avaliação da condição fisica e limitações } \\
\text { do paciente }\end{array}$ & $\mathrm{X}$ & $\mathrm{X}$ \\
\hline 2 & Elaboração de plano de tratamento & $\mathrm{X}$ & $\mathrm{X}$ \\
\hline 3 & Reabilitação - Terapia Manual & $\mathrm{X}$ & $\mathrm{X}$ \\
\hline 4 & Reabilitação - Atividade Fisica & $\mathrm{X}$ & $\mathrm{X}$ \\
\hline 5 & Utilização de VNI & $\mathrm{X}$ & \\
\hline 6 & $\begin{array}{c}\text { Manter o condicionamento fisico (após } \\
\text { recuperação de 80\% do quadro } \\
\text { funcional) }\end{array}$ & & \\
\hline
\end{tabular}

Fonte: Dados da pesquisa (2021). 
Os dados presentes no quadro, faz o levantamento das principais atividades desenvolvidas durante o atendimento domiciliar, conforme evidenciado nesse estudo. Pode-se observar que em determinadas ações ambas as profissões podem atuar, outras a atribuição é determinada para apenas um dos profissionais, após apuração das permissões estabelecidas pelos conselhos federais de cada profissão.

Figura 2 - Porcentual avaliando as atribuições estabelecidas no Quadro 1.

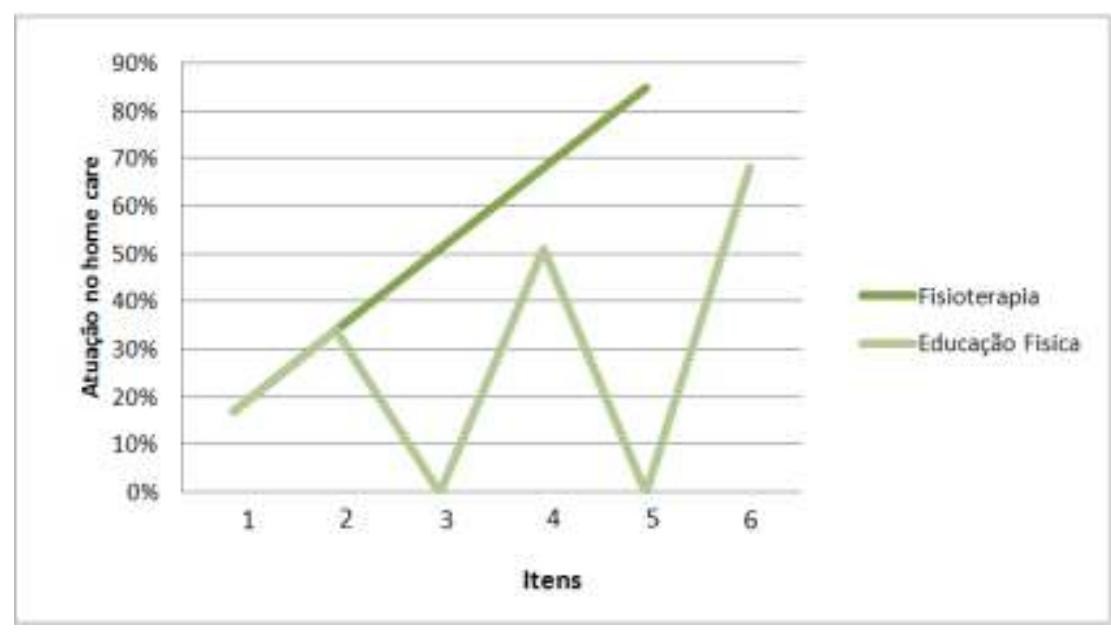

Fonte: Dados da pesquisa (2021).

De acordo com a Figura 2, no qual faz análise quantitativa dos dados fornecidos no Quadro 1, podemos analisar que, apesar de ambas as profissões contribuírem para o atendimento domiciliar do perfil de paciente analisado nesta pesquisa, a predominância e maior porcentual de permissões é dos fisioterapeutas. Os itens citados no Quadro 1 somam o total de 100\% e conforme ánalise de atribuições Fisioterapia possui $89 \%$ de qualificação e responsabilidade para o atendimento domiciliar, enquanto a Educação Física fica em $69 \%$.

\section{Discussão}

Os tipos de coronavírus conhecidos até o momento são: alfa coronavírus HCoV-229E e alfa coronavírus HCoVNL63, beta coronavírus HCoV-OC43 e beta coronavírus HCoV-HKU1, SARS-CoV (causador da síndrome respiratória aguda grave ou SARS), MERS-CoV (causador da síndrome respiratória do Oriente Médio ou MERS) e SARS-CoV-2, um novo coronavírus descrito no final de 2019 após casos registrados na China. Este provoca a doença chamada de Covid-19. (Brasil, 2021).

Os sintomas pós-Covid-19 mais frequentes são tosse, febre baixa e fadiga, mas podem incluir também dispneia, dor torácica, dor de cabeça, dificuldades neurocognitivas e tromboembolismo. (Gastaldi, 2021). De acordo com Santana, et al (2021): A reabilitação individualizada desses pacientes e o treinamento de familiares realizado no ambiente domiciliar permitem o uso dos recursos disponíveis em cada lar com as adequações necessárias em um ambiente reconhecido e de vivência do paciente, Finalmente, existem atualmente ferramentas tecnológicas que permitem a monitorização, a prescrição e o controle à distância das atividades de reabilitação, sendo aprovadas pelos conselhos federais devido ao cenário pandêmico que vivemos.

O acompanhamento multiprofissional é recomendado durante o período de reabilitação para potencializar a adesão e resultados (Spruit Et al. 2020; Sheehy et al. 2020; Jimeno-Almazan et al. 2021). A fisioterapia e a educação física atuam com o 
objetivo de reestabelecer a funcionalidade global do paciente, pois, conforme observado em relação as causas principais do Covid-19, vemos que as disfunções ocorrem principalmente no sistema respiratório, muscular e neurológico.

A recuperação dos efeitos respiratórios agudos, de acordo com a Classificação Internacional de Funcionalidade, Incapacidade e Saúde (CIF) será necessária à reabilitação complementar com um fisioterapeuta, que irá avaliar e montar um plano de tratamento específico para cada paciente, para uma melhor recuperação. (Sheehy, 2020).

Filho (2021) argumenta que: "os profissionais de educação física apresentam grande importância no trabalho multiprofissional na recuperação de paciente com Covid 19, podem monitorar a prática correta das atividades e exercícios físicos desses pacientes, com indicação dos cuidados e analisando os limites dos exercícios praticados, promovendo treinamentos para melhoria do desempenho diário, condicionamento físico e bem-estar" A inclusão do profissional de educação física tem a finalidade de manter e recuperar a saúde daquele paciente, podendo atuar juntamente com a fisioterapia na reabilitação, para indicar a melhor dosagem de exercícios para melhoria do caso clínico do paciente.

Os métodos não invasivos utilizados no tratamento desses pacientes é permitido e regulamentado para os fisioterapeutas de acordo com o acordão $\mathrm{N}^{\circ} 473$, de 20 de maio de 2016 para os profissionais com qualificação em terapia intensiva, para os profissionais de educação física não há evidências quanto a liberação para uso.

Conforme o avanço da COVID-19 a busca pela saúde tem crescido, bem como a prática de tais exercícios por uma parte da população (exercícios funcionais adaptados ao ambiente domiciliar) (França et al, 2020), onde Souza et al (2020) destaca que nesse contexto, a atividade física supervisionada pelo profissional de educação física e pela atuação do fisioterapeuta na reabilitação, serão de suma importância para esses indivíduos conseguirem retornar a sua vida normal e a funcionalidade seja garantida.

\section{Considerações Finais}

Após a análise realizada nesta revisão, foi possível identificar que ambas as profissões podem contribuir com a reabilitação pós covid-19 e que possuem formas de tratamento distintos. O principal objetivo desta revisão foi identificar qual a contribuição feita pelos profissionais e qual são as atribuições aprovadas pelos conselhos federais, pois mesmo que o profissional tenha conhecimento sobre determinada técnica, o mesmo poderá utilizar apenas quando permitido pelo conselho. Assim, o uso da VNI por exemplo, é regulamentado apenas para o fisioterapeuta para utilização em atendimentos, além de outras técnicas no qual o educador físico, apesar de possuir domínio semelhante quanto a anatomia e fisiologia humana, é autorizado apenas a realizar atividades físicas coma modalidade aeróbica, de fortalecimento muscular e flexibilidade em diversas modalidades para prevenir doenças e promover qualidade de vida. Foi evidenciado também sobre a importância do atendimento multidisciplinar no atendimento desses pacientes pós Covid-19.

É importante destacar que o quantitativo de conteúdo sobre a profissão de Educação Física no atendimento domiciliar no qual é bastante limitado, a predominância no atendimento de acordo com a análise feita é da fisioterapia, entretanto, os educadores físicos estão presente nesse campo, teria grande contribuição o desenvolvimento de pesquisas sobre os mesmos nessa área de atuação, pois enriquece o conhecimento populacional sobre qual profissional é o mais qualificado para executar a reabilitação.

\section{Agradecimentos}

O desenvolvimento deste trabalho contou com a ajuda de diversas pessoas, dentre as quais eu agradeço: aos deuses budistas e ao Gohonzon que são minha principal fonte de fé e que me sustentaram para concluir meu trajeto acadêmico. A minha querida avó que hoje não está mais presente neste plano, mas durante sua vida me incentivou e me ajudou na rotina diária de faculdade e trabalho, aos meus pais, pois este artigo não estaria sendo finalizado se não fosse o apoio e ajuda durante 
todo o percurso, principalmente nos cuidados com a minha filha nas horas em que precisei estar ausente, a minha filha, Safira Cardoso, que é a minha fonte de energia, o motivo no qual eu persistisse até o fim, pois quero mostrar a ela que podemos sim conquistar nossos objetivos e alcançar nossos sonhos. Agradeço imensamente aos meus professores que durante esses anos nos incentivaram a batalhar para conclusão do curso e por todo conhecimento que adquiri, em especial a 3 professores que são uma grande inspiração profissional para mim Edvaldo Alves, Jeronice Rodrigues e Natália Gonçalves. Muito obrigrado a todos que contribuiram durante esse trajeto.

\section{Referências}

Brasil (2013). Código de ética e deontologia da Fisioterapia. Conselho Federal de Fisioterapia e Terapia Ocupacional (COFFITO).

Brasil (2015). Código de Ética dos Profissionais de Educação Física registrados no Sistema CONFEF/CREFs. Conselho Federal de Educação Física. https://www.confef.org.br/confef/resolucoes/381.

Brasil (2016). Acordão $N^{\circ} 473$ - Dispõe sobre o papel do Fisioterapeuta em relação ao procedimento de montagem e/ou toca de circuitos dos ventiladores mecânicos. Conselho Federal de Fisioterapia e Terapia Ocupacional (COFFITO).

Brasil (2021). Boletim Epidemiológico Especial: Doença pelo novo coronavírus. Ministério da Saúde.

Brasil (2021). Coronavírus: o que você precisa saber e como prevenir o contágio. Ministério da Saúde.

Brasil (2021). Exercícios físicos auxiliam na reabilitação de recuperados da Covid-19. Revista Educação Física. https://www.confef.org.br/confef/comunicacao/revistaedf/4699.

Brasil (2021). Informações rápidas sobre VNI, Oxigenoterapia, Prona Espontânea e Exercícios respiratórios na COVID 19. Associação Brasileira de Fisioterapia Cardiorrespiratória e Fisioterapia em Terapia Intensiva (ASSOBRAFIR). https://assobrafir.com.br/covid-19/

Brasil (2021). Recomendação para avaliação e reabilitação pós covid-19. Associação Brasileira de Fisioterapia Cardiorrespiratória e Fisioterapia em Terapia Intensiva (ASSOBRAFIR). https://assobrafir.com.br/covid-19/.

Brasil (2021). Ventilação não- invasiva ou cânula nasal de alto fluxo no âmbito domiciliar. Associação Brasileira de Fisioterapia Cardiorrespiratória e Fisioterapia em Terapia Intensiva (ASSOBRAFIR). https://assobrafir.com.br/covid-19/.

Cássio Magalhães da Silva e Silva et al. Evidence-based physiotherapy and functionality in adult and pediatric patients with Covid-19. Journal of Human Growth Devvelopment 2020, 30(1):148-155.

Filho. A. L. M (2021). A Contribuição do profissional de educação física em equipe multiprofissional para recuperação de pacientes pós covid 19. Revista de Administração do CESMAC. Vol. 10.

França E. F., Macedo, M. M., França T. R., Cossote, D. F., Gonçalves, L., Santos P. A.C., \& Miyake, G. M (2020). Triagem de saúde para participação nos programas de exercício físico pós-pandemia de COVID-19: uma ação necessária e emergente ao profissional de educação física. Internacional Journal of Medicine and Health.; 3:1-4

Gastaldi, A. C. (2021). Fisioterapia e desafios da Covid-19. Revista Fisioterapia Pesquisa.

Goldman-Israelow B (2021). Coronavirus replication cycle (template).

Jimeno-Almazan, et al. (2021). Post-Covid-19 Syndrome and the Potential Benefits of Exercise. International Journal of Environmental Research and Public Health.

Menegatti, A. P. L., Fantin, R. A. B., \& Bernardes Júnior, L. (2021). Influência do Atendimento Fisioterapêutico Home Care em Idosos Pós Covid-19. Revista Ibero-Americana de Humanidades, Ciências E Educação, 7(8), 318-332. https://doi.org/10.51891/rease.v7i8.1888.

Santana, A. V., Fontana, A. D., \& Pitta, F. (2021). Reabilitação pulmonar pós-COVID-19. Jornal brasileiro de Pneumologia, 47(1).

Sheehy, L. M. (2020). Considerations for Postacute Rehabilitation for Survivors of Covid-19. JMIR Public Health and Surveillance.

Souza, M. O. de, Silva, A. C. S. e, Almeida, J. dos R., Santos, J. F. M., Santana, L. F., Nascimento, M. B. C., \& Souza, E. C. de. (2020). Impactos da COVID19 na aptidão cardiorrespiratória: exercícios funcionais e atividade física. Revista Brasileira de Atividade Física \& Amp; Saúde,25, 1-5. https://doi.org/10.12820/rbafs.25e0171

Spruit, M. A., et al. Covid-19: Interim Guidance on Rehabilitation in the Hospital and Post-Hospital Phase from a European Respiratory Society- And American Thoracic Society-Coordinated International Task Force. European Respiratory Journal 56 (6). 2020.

Toledo, A. M. (2021). Exercícios Físicos domiciliares e práticas de educação em saúde: estratégias de enfrentamento durante a pandemia covid-19. Universidade de Brasília.

World Health Organization (2020). Coronavírus Disease (COVID-19). 